\title{
Prospects for the development of the theory of designing optimal cycles of machining in a multidimensional space of control parameters
}

\author{
Aleksandra Akintseva ${ }^{1, *}$ and Pavel Pereverzev ${ }^{2}$ \\ ${ }^{1}$ South Ural State University, Department of Engineering and Technology, 454080 Chelyabinsk, Lenin Avenue 76, Russian Federation \\ ${ }^{2}$ South Ural State University, Department of Automated Engineering Technology,76, Lenin Avenue, Chelyabinsk, 454080, Russian \\ Federation
}

\begin{abstract}
In the engineering industry there are still no domestic systems of designing efficient automatic cycles for CNC machines because of the lack of a theory of their design. The article describes the approach to the development of the designing theory of optimal automatic cycles, covering all types of machining operations. The basis of this theory is a mathematical model for controlling the process of metal removal, adequately describing the process of metal removal using the whole complex of control parameters as with all possible combinations of the main technological factors as and in the wide ranges of their allowable variation. The dynamic programming method (DPM) is used as a mathematical method of optimization; it can be used for complex optimization of the processing cycle in the multidimensional space of control parameters with any number of constraints verified at each step of a directed enumeration of calculation options. As a result, it becomes possible to optimize an unlimited number of control parameters for all types of machining operations performed on $\mathrm{CNC}$ machines
\end{abstract}

\section{Introduction}

One of the main problems of modern automated mechanical engineering is the low productivity of operations of billets machining on CNC machines, especially in serial and small-scale production when there are no test billets. The reason for this problem is the lack of design systems for optimal stepwise processing cycles used in control programs for $\mathrm{CNC}$ machines. The solution of the problem above is connected with great difficulties, because the ideal variant involves simultaneous optimization of two dozen of control parameters. For example, in case of internal grinding operation with three-stage machining cycle it's necessary to set six feeds for each stage of the cycle (three radial and three axial) and three values of the part of the removed allowance at each stage. As for mode parameters, the rotation frequency of the circle and the billet must be also set. Parameters of the circle characterization and technological adjustment also refer to the control parameters. Therefore, when designing the operation, the technologist sets: grain material, bundle material, graininess, hardness and structure of the circle, geometric parameters of the circle, overtravel and other parameters. And all these parameters above should be optimal and they should be selected simultaneously. It also should be noted that the choice of the optimum parameters of the stepwise cycle should be made by the technologist considering various technological limitations inherent in the projected machining operation, namely: limitations on the requirements of the operational sketch (tolerances of diameter, shape and location, required roughness and hardness of the machined surface), machine limitations (ranges of feed, rotational frequencies and drive powers, achievable processing accuracy, rigidity of the technological system), limitations on the active control device (number of stages, measurement error), limitations on the cutting tool (design, geometry, strength, wear resistance), limitations on the batch of the processed billets (allowance fluctuation, blunting of the cutting tool), etc.

In fact, in the absence of automated systems for designing machining cycles for $\mathrm{CNC}$ machines the technologist must simultaneously set dozens of optimal control parameters considering various technological limitations. It's a complex scientific and technical task which is naturally beyond the power of any technologist. Therefore, in real production technologists set processing cycles based on existing production statistics and outdated general machine-building standards for cutting modes of the 90 -ies of the edition, intended more for rationing operations $[1,2$, etc.]. It should be noted that the standards are based on the domestic production statistics and reflect the average level of cutting regimes in the country in the 80 s at the then existing machine tool park.

Therefore, regulatory recommendations are neither optimal nor effective for modern $\mathrm{CNC}$ machines and they do not provide high-performance processing. According to the regulations, at first the technologist

\footnotetext{
* Corresponding author: popovaav@susu.ru
} 
selects the parameters of the cutting tool on the basis of some recommendations. Then, depending on the range of diameters and processing lengths, the technologist selects the normative base cycle and the rotational speed of the part for fixed basic regulatory conditions. If the actual conditions in production differ from normative ones then the technologist must somehow make a correction of the regime parameters of the cycle or cutting tool. The results of such changes in the cycle and parameters of the cutting tools are poorly predictable due to the lack of verification models. Since such a normative cycle carries a high risk of getting a reject, for the guarantee, the technologist additionally underestimates the performance parameters while reducing the cycle productivity.

As a result, optimization of machining cycles for CNC machines is simply impossible in such production conditions. This situation is of a mass nature in engineering. Therefore, CNC machines are used inefficiently and machining operations are performed according to our estimation with a performance which is understated up to two times.

It should be noted that there is insufficient attention given to the problems of the theory of designing optimal machining cycles for $\mathrm{CNC}$ machines. An overwhelming number of researchers are studying the particular aspects of the problem of designing optimal control trajectories [3-8, etc.]. The greatest attention is paid to modeling the cutting force and modeling the technological limitations (on accuracy, roughness, defect layer, wear and tool persistence), affecting the performance of operations for particular conditions. The results of these studies are particular recommendations for partial optimization of cutting modes for a limited number of constraints in a narrow range of their variation. There are very few works aimed at developing the theory of constructing optimal control programs for $\mathrm{CNC}$ machine tools that simultaneously consider a set of technological limitations (on parameters of the billet, machined surface, machine, cutting tools, machine control system, unstable conditions of production organization and course of the processing process).

Thus, the domestic mechanical engineering has to solve actual problem of increasing the productivity of machining operations performed on $\mathrm{CNC}$ machines.

\section{Development of the automated design system of optimal cycles of machining in a multidimensional space of control parameters}

The theory of optimal step cycles designing has been developed to solve the problem above and to increase the effective use of CNC machines in machining operations based on the following three components:

1. Mathematical model that adequately describes the process of metal removal using the entire complex of control parameters, as with all possible combinations of basic technological factors as and in wide ranges of their permissible variation. For example, on fig. 1 three-stage cycle with circular plunge grinding is shown. The program feed $S_{\Pi}$ is set from the machine control panel. The change of $S_{\Pi}$ in the three-stage cycle during the grinding process is performed according to the commands of the active control device (ACD) depending on the remaining part of the allowance. The actual feed $S_{\Phi}$, which occurs when grinding the machined surface (fig. 1) and a result of which the cutting force arises and varies depending on the rigidity of the technological system and other numerous parameters. The $S_{\Phi}$ value is controlled by a stepwise switching of the program feed $S_{\Pi}$.

2. Mathematical models of the set of technological constraints described above. Interrelation between the stepwise cycle of the radial feed and technological constraints complex is shown on fig. 1. Most of the limitations cut off the range of allowable values of the actual feed $S_{\Phi}$. The limitation on the range of feeds on the machine cuts off the range of allowable values of the program feed $S_{\Pi}$.

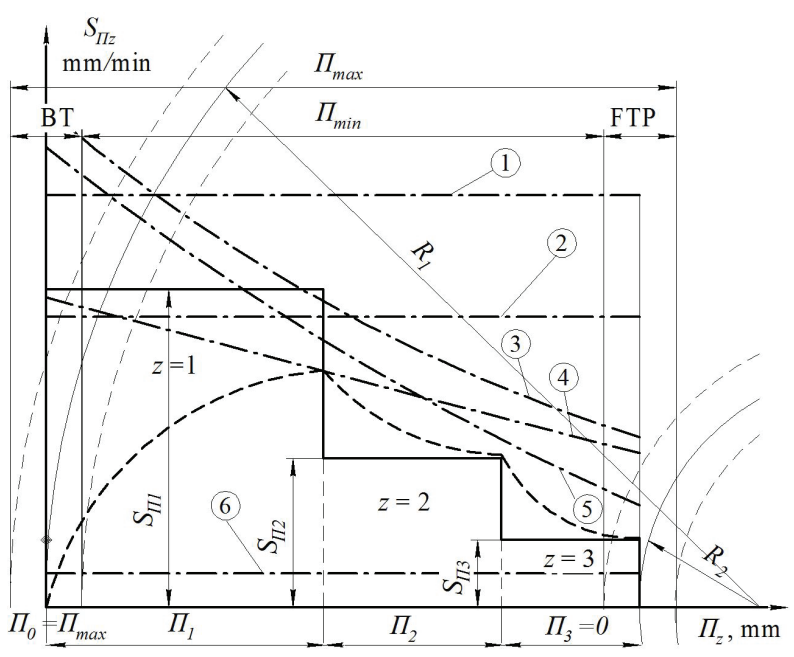

Fig. 1. Range of allowable values of the actual feeds of the three-stage circle plunge grinding: $\mathrm{S} \Phi \mathrm{z}$ - actual feed, $\mathrm{mm} / \mathrm{min}$; 1 - limitation on the maximum feed speed; 2 - limitation on the circle falling; 3 - limitation on the dimensional accuracy; 4 - limitation on the burn; 5 - roughness limitation; 6 limitation on the maximum feed speed; BT - billet tolerance; FPT - finished part tolerance;

3. Mathematical method of optimization based on the dynamic programming method (DPM). The process of optimizing the processing cycle is to maximally approximate the trajectory of the $S_{\Phi}$ to the active limit of constraints by stepwise switching of the program feed of the $S_{\Pi}$ (fig. 1). The more the number of stages of $S_{\Pi}$ switching, the closer the trajectory can be approximated to the $S_{\Phi}$ limit constraints and the higher performance of processing cycle can be achieved. Thus, the «stepless» cycle, i.e. cycle with an infinite number of steps will be the most productive. However, in practice, cycles which have no more than five steps are used because the addition of each new step increases cycle productivity by a fraction of a percent. In addition, as the number of steps increases the complexity of setting up the process of processing and its realization increase. Therefore the ACD has a limited number of feed switching stages. Since it is necessary to simultaneously optimize several 
dozens of control parameters (listed above), a new method of applying the DPM has been developed for their optimization; it allows designing optimal step cycles in a multidimensional space of control parameters. The advantage of this new technique is its insensitivity to the number of optimized control parameters and the lack of requirements for their properties by differentiability, nonlinearity and complexity of their mathematical models. For example, the optimized cycle of radial fed for round external grinding is a stepwise linear function (fig. 1), which can not be described in the form of a single formula and this function does not have the property of differentiability. In addition, the new optimization technique is also insensitive to the number and properties of the technology constraint models and does not require previous creation of the control parameters limits. For example, the roughness constraint of the processed surface is specified and valid only for the finite point of the cycle (i.e. the roughness should be obtained at the end of the machining cycle). Therefore, this limitation can not be calculated and carried out in advance throughout the processing cycle, although it is conventionally drawn in the graph fig. 1. Similarly, the constraint on the processing accuracy also acts only at the end of the cycle and it can not be calculated and drawn on the graph throughout the cycle.

Creation of the computer-aided design system is suggested to lead by the following typical algorithm:

1) creation of a mathematical model for controlling the process of metal removal and control of a complex of control parameters, productivity of the machining operation;

2) creation of mathematical models of control parameters constraints;

3) adaptation of the dynamic programming method for optimization of control cycles in a multidimensional space of control parameters;

4) development of software for the design system of optimal processing cycles.

Approaches to stages 1 and 2 are described in detail in a number of works on the example of round external and internal grinding [9-10, etc.]. Let us consider the third stage in more detail as it is the most difficult and little studied. Optimization of the processing cycle is carried out with the help of DPM in analogy with the optimization of the transport task when it is necessary to find the optimal route on a branched network of roads from the starting point to the final [11].

As the DPM refers to discrete methods then the partial optimization of the cycle for two control parameters (program feed and distribution of allowance for the cycle stages) is carried out on the coordinate grid «Program feed $S_{\Pi}$ - Allowance $\Pi$ (fig. 2). To obtain the coordinate grid the axes of the graph are divided into a plurality of discrete values (discrete). The procedure for optimizing the DPM is carried out by the coordinate grid notes. Therefore, the smaller the grid cell (i.e. the more discrete) the higher the accuracy of the optimal solution but the higher the complexity of the optimization due to the large number of nodes. If the optimization of the cycle is carried out without considering the limitations on the number of permissible feed switching steps then, as a result of optimization, we get the optimal «stepless»» (code name) cycle because of a huge number of discrete a stepless cycle is obtained; this cycle due to the discreteness of DPM will have many small steps the number of which depends only on the discreteness of the coordinate grid. On fig. 2 the stepless cycle on the coordinate grid with the number of discrete $10 \times 20$ is shown. If the optimization of the cycle is carried out considering the limitation on the number of permissible feed switching stages, then for example, for a three-step cycle we obtain the optimal three-step cycle as a result of optimization (fig. 2), in which the program feeds and allowances for each step are optimal.

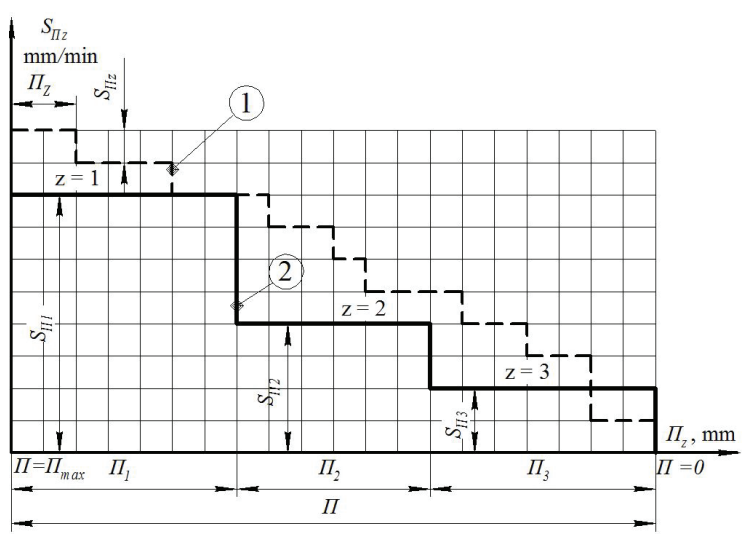

Fig. 2. Coordinate grid «Program feed SП - Allowance П» with a number of discrete 10x20: 1 - «stepless» cycle; 2 three-step cycle;

First let us consider the simplest case when the limitation on the number of switching steps is not considered. In accordance with the DPM optimization procedure in all grid notes (fig. 3) the information cell with the coordinates $[n, m]$ is placed, where $n$-discrete number of allowance, $m$ - discrete number of program feed. In the process of optimizing DPM between two grid notes it is necessary to specify the travel time $\tau_{x}$, necessary to move from one node to another. To know the travel time between two nodes the process of metal removal is simulated; it is equal to the allowance discrete at the feed levels depending on the coordinates of the nodes between which the stroke is made. As several moves can be made to one node the best one is selected from all possible moves which ensures the minimum accumulated time. The optimal (minimal) time of reaching the state $T_{n, m}$ is written to the information cell and in the variable $m^{*}$ the coordinate of the node from which the optimal move is made is memorized (fig. 3). After applying the standard DPM procedure - reverse move, we get the optimal trajectory of «stepless» cycle (fig. 2), in which the values of the program feed $S_{\Pi}$ and corresponding to them discrete allowances $\Pi_{z}$ are optimal. 


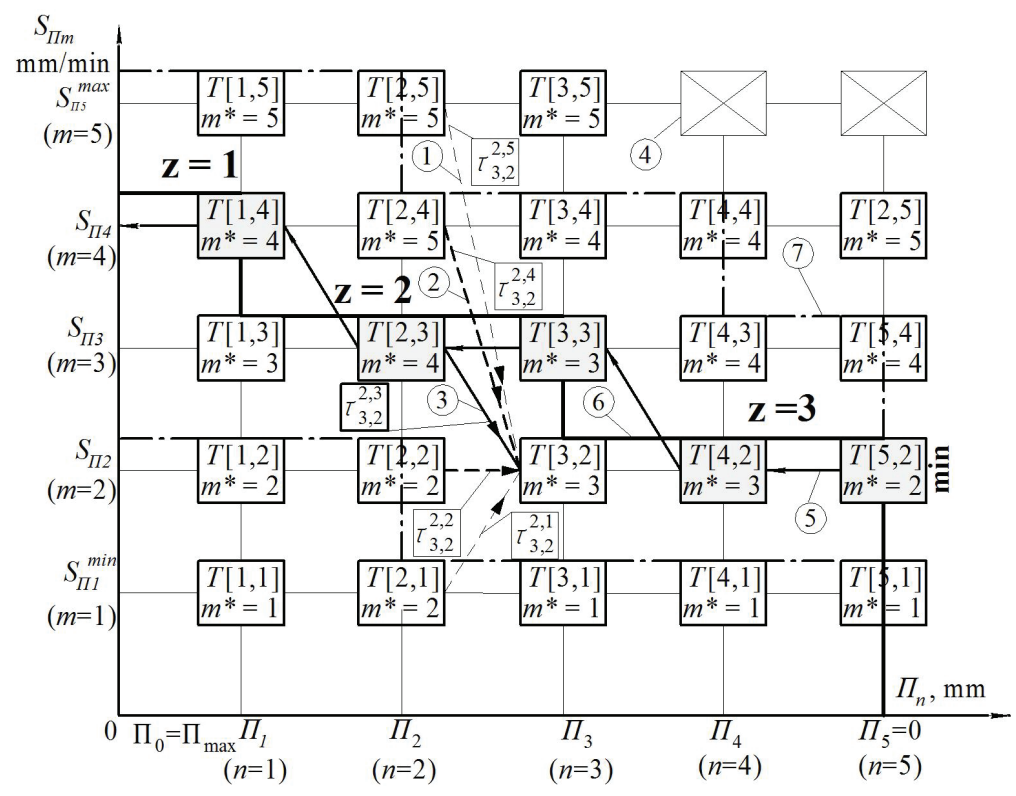

Fig. 3. Coordinate grid for the optimization of the round plunge grinding cycle by dynamic programming method: $\mathrm{T}$ [m, $\mathrm{n}]-$ optimal time of reaching the state $\boldsymbol{T}_{\boldsymbol{n}, \boldsymbol{m}} ; 1$ - competing moves; 2 -admissible moves; 3 -optimal move; 4 -information cells eliminated from the calculation; 5 - reverse move procedure; 6 - trajectory of optimal control; 7 - possible cycles;

As the «stepless» cycles are not used in production practice let us consider the optimization of the cycle considering the limitation on the number of cycle stages. In this case, the structure of the information cell is complicated because the number of stage is added. An example of the information cell for the three-step cycle is given on (fig. 4).

\begin{tabular}{|c|c|c|}
\hline $\boldsymbol{z}$ & $\boldsymbol{T}_{n, \boldsymbol{m}, \boldsymbol{z}}$ & $\boldsymbol{m}^{*}(\boldsymbol{n}, \boldsymbol{m}, \boldsymbol{z})$ \\
\hline$z_{\max }$ & $T_{n, m, z_{\max }}$ & $m^{*}\left(n, m, z_{\max }\right)$ \\
\hline$\ldots$ & $\ldots$ & $\ldots$ \\
\hline$z+1$ & $T_{n, m, z+1}$ & $m^{*}(n, m, z+1)$ \\
\hline$z$ & $T_{n, m, z}$ & $m^{*}(n, m, z)$ \\
\hline$z-1$ & $T_{n, m, z-1}$ & $m^{*}(n, m, z-1)$ \\
\hline$\ldots$ & $\ldots$ & $\ldots$ \\
\hline 1 & $T_{n, m, 1}$ & $m^{*}(n, m, 1)$ \\
\hline
\end{tabular}

Fig. 4. Structure and content of the information cell at optimization considering the limitation on the number of cycle stages: $z$ - stage number of the cycle; $T_{n, m, z}-$ optimal (minimal) time of reaching the state; $m^{*}$ - memorized value of the coordinate grid note from which the optimal move is made.

In the process of optimization considering the limitation on the permissible number of program feed switching the following principle should be observed: if a move with program feed level is made, then the stage number $z$ increases by one, otherwise the stage number $z$ does not change (fig. 5). This principle remains in the case of reverse move [12-13].

After the DPM reverse move the partially optimal three-step cycle will be obtained (fig. 2), in which only two control parameters are optimized: program feed and distribution of the removed allowance part along the cycle stages.

Let us consider an even more complicated case when another control parameter is optimized. For example, let us optimize the processing cycles for two feeds (radial and axial) depending on the remaining part of the allowance. Optimization is carried out on the coordinate grid « Program feed $S_{\Pi}-$ Allowance $\Pi$ ». Thus, two control parameters are considered in the DPM system. The third control parameter - program axial feed $V_{S o c}$ will be considered in the information cell structure (fig. $6)$.

\begin{tabular}{|c|c|c|c|c|}
\hline$V_{\text {Socb }}$ & $z$ & $T_{n, m, b, z}$ & $\begin{array}{c}m^{*} \\
(n, m, b, z)\end{array}$ & $\begin{array}{c}b^{*} \\
(\boldsymbol{n}, \boldsymbol{m}, b, z)\end{array}$ \\
\hline \multirow{3}{*}{$\begin{array}{l}V_{S o c 2} \\
(b=2)\end{array}$} & $z=3$ & $T_{n, m, 2,3}$ & $\begin{array}{c}m^{*} \\
(n, m, 2,3)\end{array}$ & $\begin{array}{c}b^{*} \\
(n, m, 2,3)\end{array}$ \\
\hline & $z=2$ & $T_{n, m, 2,2}$ & $\begin{array}{c}m^{*} \\
(n, m, 2,2)\end{array}$ & $\begin{array}{c}b^{*} \\
(n, m, 2,2)\end{array}$ \\
\hline & $z=1$ & $T_{n, m, 2,1}$ & $\begin{array}{c}m^{*} \\
(n, m, 2,1)\end{array}$ & $\begin{array}{c}b^{*} \\
(n, m, 2,1)\end{array}$ \\
\hline \multirow{3}{*}{$\begin{array}{l}V_{\text {Soc } 1} \\
(b=1)\end{array}$} & $z=3$ & $T_{n, m, 1,3}$ & $\begin{array}{c}m^{*} \\
(n, m, 1,3)\end{array}$ & $\begin{array}{c}b^{*} \\
(n, m, 1,3)\end{array}$ \\
\hline & $z=2$ & $T_{n, m, 1,2}$ & $\begin{array}{c}m^{*} \\
(n, m, 1,2)\end{array}$ & $\begin{array}{c}b^{*} \\
(n, m, 1,2)\end{array}$ \\
\hline & $z=1$ & $T_{n, m, 1,1}$ & $\begin{array}{c}m^{*} \\
(n, m, 1,1)\end{array}$ & $\begin{array}{c}b^{*} \\
(n, m, 1,1)\end{array}$ \\
\hline
\end{tabular}

Fig. 6. Structure of the information cell at the cycles optimization for two feeds depending on the part of the removed allowance.

Optimization of control parameters and finding the trajectory is carried out according to the same principles as shown on fig. 5. The result of the optimization will be two optimal trajectories for the radial and axial feeds with the same number of stages and distribution of the allowance in the stages. Similarly, any number of optimized control parameters can be added including parameters of the circle characteristics and technological adjustments. As a result it becomes possible to optimize an unlimited number of cycle control parameters if there is an adequate model of the process of controlling metal removal productivity. 


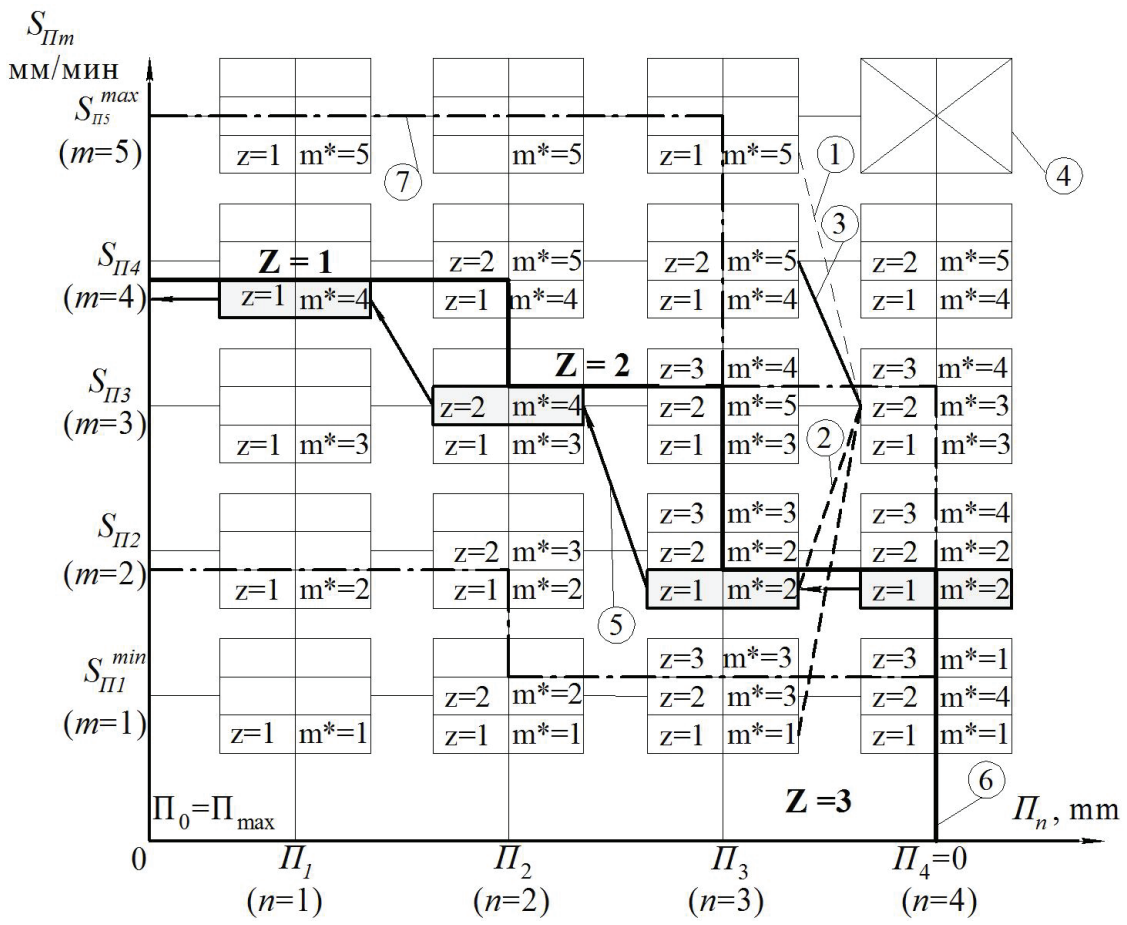

Fig. 5. Reverse move procedure considering the limitation for the admissible number of program feed switching: 1 - competing moves; 2 -admissible moves; 3 -optimal move; 4 -information cells eliminated from the calculation; 5 - reverse move procedure; 6 - trajectory of optimal control; 7 - possible cycles.

The solution to the problem of productivity increasing for all types of machining operations performed on $\mathrm{CNC}$ machines is beyond the power of one department because for all types of operations it is necessary to develop processes models and constraint models with subsequent adaptation of the DPM for optimization of the control cycles in the multidimensional space of control parameters. It is necessary to make a lot of analysis which will require modern laboratories with test benches for all types of operations. The solution of such a problem is possible only within the framework of the implementation of a single large project on the basis of a constantly operating technology center in which technologists, programmers, specialists in CNC systems, scientists with knowledge of various types of processing and related areas of scientific knowledge should participate. With the processes and constraints models it is possible to start creating software for optimization the processing cycles and control programs. Software development consists of known typical stages:

- analysis of requirements for the created software to determine the key goals and objectives of the final product and formation of the technical task;

- software design, choice of tools and operating system;

- coding software on the basis of the chosen programming language;

- testing and debugging of software allowing to eliminate programming errors and achieve the ultimate goal - full-featured work of the developed program;

- implementation of the software in operation and its further support is the final stage of the development.

\section{Conclusion}

1) developed theory of optimal automatic cycles designing:

- covers all types of machining operations (including turning, milling, grinding, drilling) performed on $\mathrm{CNC}$ machines;

- based on the development of the DPM application for the complex optimization of the processing cycle in the multidimensional space of control parameters with any number of constraints verified at each step of a directed search of variants calculation.

2) software structure for optimizing DPM cycles is united for different types of machining;

3 ) in general for the entire automated engineering industry it is necessary to develop over a hundred techniques and software products for the design of optimal cycles what will raise the machining productivity in the machine-building industry by two or more times on $\mathrm{CNC}$ machines;

4) task of developing hundreds of techniques and software products for designing optimal cycles is beyond the power of a one team. Its solution requires mobilization of all scientific and technological potential of the country under the management of a national technology center with a developed network of research laboratories.

\section{References}

1. Engineering industry standards of time and the cutting modes for rationing of the works carried out on the 
universal and multi-purpose machines with numerical program control. Part II. Standards of the cutting modes (1990)

2. D.V. Ardashev, D.E. Anelchik, G. I. Butorin and etc., Modes of cutting for work performed on grinding and finishing machines with manual control and semiautomatic machines: reference book (2007)

3. G.B. Lurye, Optimization of a cycle of grinding on the basis of adaptive management (1979)

4. V.N. Mikhel'kevich, Automatic Control of Grinding (1975)

5. Y.K. Novoselov, Dynamics of surface formation during abrasive processing (2012)

6. S.S. Saykin, P.M. Salov, T.G. Vinogradova, Innovation in the educational process 8, 61 (2010)

7. A.A. Nikolaenko, Technology of machine building $\mathbf{5}$, 57 (2011)

8. D.Yu. Pimenov, Journal of Friction and Wear 1, 643 (2015)

9. P.P. Pereverzev, A.V. Akintseva, Russian Engineering Research 4, 23 (2016)

10. P.P. Pereverzev, A.V. Akintseva, Bulletin of the South Ural State University. Ser. Mashinostroenie 2, 61 (2016)

11. R. Bellman, Dynamic programming (1960)

12. P.P. Pereverzev, A.V. Akintseva, Russian Engineering Research 1, 11 (2017)

13. P.P. Pereverzev, A.V. Akintseva, Russian Engineering Research 2, 13 (2017) 